\title{
Improved estimation of surgery duration using exponential distribution for the elective surgery scheduling problem
}

\begin{abstract}
Surgery scheduling problem is complicated since the surgery duration is uncertain and a good method to estimate the surgery duration accurately is needed. Failure to do so might affect the operating room (OR) utilization and patient's waiting time. This paper uses statistical distribution for the elective patient's surgery duration to produce a good estimation of length of the surgery duration. Our aim is to maximize the sum of the urgency values assigned to each surgery. The results show that Exponential Distribution (ED) is better than Uniform Distribution (UD) in scheduling the urgency value for scheduled surgeries. ED also reduces the urgency value for the unscheduled surgeries slightly.
\end{abstract}

Keyword: Surgery scheduling problem; Heuristic; Surgery duration; Exponential disribution; Uniform distribution 